\title{
Modelo ambiental para una ciudad capital ${ }^{*}$
}

\author{
Environmental model for a capital city \\ Recibido: 24 de octubre de 2012 - Revisado: 21 de diciembre de 2012 - Aceptado: 30 de enero de 2013
}

\author{
Claudia Eugenia Toca Torres** \\ Jesús Carrillo Rodríguez ${ }^{* * *}$
}

\begin{abstract}
Resumen
A partir de una revisión de las distintas opciones para la modelación del desarrollo sostenible en su dimensión ambiental, esta investigación propone un modelo de impacto ambiental para Bogotá, usando el software Vensim PLE para modelar la polución, la carga contaminante y la contaminación de suelos. El modelo incluye un limitado número de variables endógenas, como también un número mayor de variables exógenas. Esta modelación permite anticipar la situación ambiental de la capital, a fin de soportar políticas públicas para tratar asuntos como las sanciones económicas y morales; regulaciones sobre emisiones, vertimientos y residuos; medidas ambientales y prácticas ambientalmente amigables.
\end{abstract}

\section{Palabras clave}

Modelación, polución, carga contaminante, contaminación de suelos.

\section{Abstract}

From a review of the various options for modeling a sustainable development in its environmental dimension, this research proposes a model of environmental impact for Bogota, using the Vensim PLE software to model the pollution, the pollution load and soil contamination. The model includes a limited number of endogenous variables, as well as a greater number of exogenous variables. This modeling allows us to anticipate the environmental situation in the capital, in order to support public policies for addressing issues such as economic sanctions and moral regulations on emissions, discharges and waste, environmental measures and environmentally friendly practices.

Key Words

Modeling, pollution, pollution load, soil contamination

\footnotetext{
"Este artículo es un producto del Observatorio de Políticas, Ejecución y Resultados de la Administración Pública, OPERA, del Centro de Investigaciones y Proyectos Especiales, CIPE, de la Facultad de Finanzas, Gobierno y Relaciones Internacionales de la Universidad Externado de Colombia.

*** Especialización en Gobierno, Gerencia y Asuntos Públicos. Universidad Externado de Colombia. Facultad de Finanzas, Gobierno y Relaciones Internacionales.

Correo electrónico: cleutoto@etb.net.co.

*** Doctorado en Estudios Políticos. Universidad Externado de Colombia. Facultad de Finanzas, Gobierno y Relaciones Internacionales.

Correo electrónico: jesus.carrillo@uexternado.edu.co.
} 


\section{Introducción}

No se puede negar que las empresas en calidad de organizaciones del mercado han contribuido sobremanera a impactar los recursos naturales de la capital y que es el gobierno distrital el que debe garantizar su recuperación. Sin embargo, las empresas deben minimizar dicho impacto mediante la reducción o eliminación de residuos (sólidos, líquidos, gases o humos) por tratarse de un mandato del desarrollo sostenible. Para analizar el impacto ambiental de un área geográfica han sido utilizados métodos estáticos para periodos de tiempo fijos y que permiten la relación de un limitado número de variables. Es en este sentido que los indicadores han soportado el análisis del impacto ambiental. De igual modo, se encuentran métodos dinámicos y soportados en la complejidad, cuyos resultados se determinan en función de la interacción futura de una serie de variables endógenas y exógenas. Por incorporar una amplia variedad de sujetos y fenómenos conductuales en el tiempo, la modelación y la simulación, pueden soportar adecuadamente decisiones actuales y garantizar de paso resultados futuros.

Con estas claridades, la presente investigación se soporta en el desarrollo sostenible y en los recursos naturales de uso común y en métodos analíticos dinámicos para proponer un modelo de impacto ambiental para la ciudad de Bogotá. Para cumplir con este propósito, el primer apartado describe la dimensión ambiental del desarrollo sostenible y establece que frente a la imposibilidad de las empresas para autorregular los impactos sobre los recursos de uso común (acción colectiva), la única opción posible es gobernarlos a través de políticas públicas reguladoras. La segunda sección presenta una revisión de las distintas posibilidades de modelación del desarrollo sostenible y en particular de la dimensión ambiental. El tercer apartado propone un modelo de impacto ambiental para cada uno de los recursos naturales (aire, agua y suelos) de la capital colombiana. Para terminar, se presenta el Vensim como un lenguaje gráfico para so- portar la simulación del modelo propuesto y se establece la relación del impacto ambiental con asuntos sociales como salud de la población, productividad escolar y laboral y, desde luego, bienestar social (dimensión societal).

\section{Dimensión ambiental del desarrollo sostenible}

En la definición del desarrollo sostenible (DS) como "el desarrollo que satisface necesidades del presente sin comprometer la habilidad de generaciones futuras para satisfacer sus propias necesidades" (WCED, 1987, en Duran y Paucar, 2009, p. 79), han sido identificados tres valores fundamentales: equidad intergeneracional, mantenimiento del capital natural y justicia intergeneracional. En este sentido, ecoeficiencia, ecojusticia y principio cautelar han sido temas asociados con dichos valores. El primero refiere el uso prudente y conservación de los recursos naturales; el segundo, la forma de distribución equitativa de los productos de la naturaleza dentro y entre generaciones, y el tercero, relacionado con la necesidad de adoptar un criterio de bajo riesgo sobre la toma de decisiones para evitar el daño a generaciones futuras. Una definición muy simple del desarrollo sostenible incluye las actividades que mejoran la condición humana y que pueden ser sostenidas en el tiempo. Definición de la que emergen una serie de nociones no mencionadas como precaución o administración del riesgo, ya que los resultados de muchas actividades no se conocen bajo condiciones de certidumbre; protección ambiental y preservación de recursos naturales; y condiciones humanas actuales y de generaciones futuras (Sage, 1999, p. 194).

En el marco del DS las necesidades se centran en cinco tipos de capital: natural, financiero, humano, tecnológico e institucional y de infraestructura (Sage, 1999, p. 191). De forma particular, se identifican cambios generados por las empresas sobre el capital natural: 1) transformación de la tierra y el mar mediante tala, silvicultura, pastoreo, urbanización, minería, 
pesca con red, dragado y demás; 2) alteración de los principales ciclos bioquímicos de carbón, nitrógeno, agua y químicos sintéticos; y 3 ) adición o remoción de especies mediante alteraciones o pérdidas de hábitats, caza, pesca e introducción e invasión de especies. Bajo estas circunstancias, se ha llegado a la conclusión que durante las últimas décadas los humanos se han convertido en una nueva fuerza en la naturaleza; han modificado los sistemas físicos, químicos y biológicos de la Tierra. Y si bien los resultados de dichas modificaciones no son del todo conocidos, sí podrían tener profundas implicaciones para la vida (Lubchenco, 1999 en Sage, 1999, p. 192).

Bajo una visión optimista del DS se cree en la sustitución de recursos naturales agotables; bajo una pesimista, dicha sustitución es limitada. La primera se refleja en lo que se ha denominado perspectiva débil del DS y es considerada básicamente desde el punto de vista de la extracción de recursos no renovables como los combustibles fósiles y minerales. La perspectiva fuerte, en cambio, considera que existe más que el valor total de un sistema natural, que la simple suma de valores de sus componentes y que la sustitución puede ser una consecuencia. Si el consumo de recursos se mantiene en el tiempo, los servicios básicos del capital natural deberán preservarse (Mueller, 2008, p. 217).

Un enfoque adecuado para el análisis de las interacciones entre la economía y los sistemas naturales vitales exige, para iniciar, reconocer que el DS es desarrollo que perdura y tratarlo como un proceso evolutivo. Podría, por tanto, ser asociado con la estabilidad de la evolución y las fuerzas subyacentes que conectan desarrollo y estabilidad reflejadas en el estado de cuasiequilibrio de los sistemas naturales. El cuasiequilibrio es un estado que involucra un proceso de cambio continuo con modelos o parámetros estables (Boulding, 1991, en Mueller, 2008, p. 219).

La adopción de un enfoque evolutivo en la economía ambiental obedece precisamente al concepto de DS, considerado un proceso de cambio que incluye no solo aspectos cuantitativos sino también cualitativos en un horizonte de largo plazo y una dependencia mutua de calidad ambiental y disponibilidad de recursos, por un lado, y desarrollo económico por otro. (Mulder y Van Den Bergh, 2001, p. 114). Los bajos resultados en materia ambiental y social se convierten en estándar ante el esfuerzo que implica hacer algo efectivo. Un sistema que basa sus objetivos en la realidad y que únicamente pretende mejorarla se inclina hacia una tendencia permanente por los bajos resultados. Contrariamente, un sistema al que le fijan los objetivos desde una base externa no puede negociar los resultados.

Muchos problemas ambientales importantes enfrentan incertidumbre cierta (evento con una probabilidad desconocida a veces referida como indeterminación) y riesgo (evento con una probabilidad conocida a veces referida como incertidumbre estadística). Es así que el análisis ecológico indica el rango de incertidumbre sobre el calentamiento global, los impactos potenciales de los químicos tóxicos o el posible rango de las dinámicas poblacionales de peces y algo sobre las probabilidades relativas de diferentes resultados. En la mayoría de casos, dicho análisis no está en capacidad de indicar con precisión el rango de riesgo (Costanza, Bobbi, Ostrom y Wilson, 2001, p. 10).

Conviene establecer ahora la diferencia entre los recursos de uso común y los bienes públicos, pues en ocasiones se afirma que los recursos naturales son bienes públicos. El recurso de uso común "alude un sistema de recursos (naturales o hechos por el hombre) lo suficientemente grande como para volver costoso excluir a destinatarios potenciales de los beneficios de uso" (Ostrom, 2000, p. 66).

En cuanto al uso de los recursos naturales, en 1954, H. Scott Gordon a partir de una investigación sobre la pesca concluyó que "los peces en el mar no tienen valor para 
el pescador, porque no hay ninguna garantía de que estarán esperándolo mañana si hoy no los pesca" (Ostrom, 2000, p. 27). En términos generales, quien dilata el uso de recursos gratuitos para todos, sólo encontrará que ya han sido tomados por otros. En 1968, Garrett Hardin explicó la tragedia de los comunes a partir de los pastizales y los rebaños concluyendo que "la ruina es el destino hacia el cual todos los hombres se precipitan, persiguiendo cada uno su propio interés en una sociedad que cree en la libertad de los bienes comunes" (Ostrom, 2000, p. 27).

Así las cosas, los recursos naturales siempre se verán involucrados en juegos de suma cero. Los conflictos genéticos de intereses existen entre todos los organismos vivos: los individuos luchan por incrementar su propia idoneidad a costa de individuos no relacionados en un mundo finito. La cooperación en sistemas humanos y no humanos, se da bajo condiciones específicas: el número de actores es reducido, las interacciones son repetidas y los actores son capaces de detectar engaños y castigar infractores (Costanza, Bobbi, Ostrom y Wilson, 2001, p. 10).

El asunto urgente en el mundo se centra en encontrar la mejor manera de limitar el uso de recursos naturales para asegurar su viabilidad económica a largo plazo en un mundo en el que ni Estado ni Mercado han logrado con éxito que los individuos (y las unidades productivas) mantengan un uso productivo de largo plazo de los sistemas de recursos naturales. (Ostrom, 2000, pp. 25-26). Estado y Mercado constituyen instituciones capaces de asumir compromisos y acciones con los agentes y sujetos, enmarcados en procesos de autoorganización y autogestión. Implica entonces que grupos como los sectores productivos o industrias deben orientarse hacia la solución de problemas de provisión, compromiso y supervisión institucional para garantizar los procesos mencionados. A esta denominada acción colectiva se suman los procesos de po- líticas reguladoras que igual garantizan resultados positivos para los recursos naturales. $\mathrm{La}$ presencia de la acción colectiva, desde luego, hace injustificables las políticas. Los estudios indican que un Leviatán externo es necesario para evitar la ruina en un mundo sobrepoblado y para evitar las tragedias de los comunes. Frente al fracaso de la cooperación (acción colectiva) y a la primacía de los intereses individuales sobre los colectivos, corresponde a los gobiernos el control de la mayoría de los sistemas de recursos naturales (Ostrom, 2000, p. 36).

\section{Posibilidades de modelación del desarrollo sostenible}

Los métodos clásicos usualmente refieren el Producto Interno Bruto para evaluar el grado de desarrollo de un país, no obstante, la filosofía del DS ha llevado a reconocer que el PIB no da cuenta absoluta de la calidad de vida, la polución o el uso insostenible de recursos. Si bien surge como alternativa el Indicador de Desarrollo Humano para evaluar el desarrollo en términos sostenibles, este constituye un método estático. Se plantea así la necesidad por métodos más dinámicos en el tiempo y el espacio que incorporen situaciones y fenómenos incontrolables.

El término modelo tiene diferentes significados para diferentes comunidades. Para algunos, es una réplica física o imitación; para otros, puede ser una descripción verbal/ analítica o un diagrama en bloque con etiquetas verbales (Pew y Mayor, 1998, p. 10). Los modelos son representaciones abstractas de territorios complejos, cuya utilidad radica en el soporte que brindan para la solución de problemas. Cuando se modelan sistemas ecológicos y sociales, el propósito puede variar, desde proporcionar un entendimiento general de la conducta del sistema hasta lograr aplicaciones realistas y detalladas que soporten la evaluación de políticas específicas (Costanza, et al., 2001, p. 27). 
Entre las razones por las que resulta útil la construcción de modelos ambientales, destacan: modificar el estado del sistema porque existe un problema [ambiental] por lo que la serie histórica no tiene continuidad; comparar el comportamiento del modelo y la realidad; y aumentar el conocimiento acerca del sistema estudiado y crear un instrumento útil para el análisis de políticas. En la medida que el modelo ofrezca una salida coherente con el pasado y la situación actual, se podrá simular el impacto de las políticas o decisiones que lleven a la solución del problema. Por ejemplo, los enfoques sistémicos de modelación de los sistemas de agua en las ciudades han aumentado la habilidad para analizar un rango más amplio de impactos. Dichos modelos ayudan a incrementar e integrar el conocimiento sobre el recurso a cuantificar y medir impactos de las distintas estrategias y a soportar sus procesos de decisión y gestión operativa. Muchas de las evaluaciones basadas en modelos se centran primordialmente en los impactos ambientales y no incluyen un tratamiento amplio de asuntos económicos (Sahely y Kennedy, 2007, p. 551).

En los sistemas ambientales, la no linealidad y los intervalos espaciales y temporales son comunes. Bajo estas condiciones, la exigencia es por enfoques de modelación que identifiquen, recopilen y relacionen la información relevante para entender dichos sistemas y que garanticen consenso y guíen la toma de decisiones (Costanza, et al., 2001, p. 21).

Algunos autores han interpretado la interrelación sistémica de las cuatro áreas que agrupan los principios del desarrollo sostenible: ambiente, equidad, participación y largo plazo. (Duran y Paucar, 2009, pp. 79-80). Es así que para efectos de modelación, el desarrollo sostenible igualmente ha sido agrupado en cuatro asuntos: ambientales (recursos nacionales, polución ambiental, uso de tierra, biodiversidad), económicos (finanzas, servicios públicos, infraestructura, empleo), sociales (inclusión, seguridad física y social, salud, comunidad) e institucionales (governance, justicia, sistema ético).

Los enfoques de modelación del DS han sido agrupados en macromodelos, micromodelos, mesomodelos y modelos integrados. Ningún modelo es considerado correcto, y el mejor enfoque depende de la naturaleza del problema a ser resuelto. El macromodelo considera interacciones entre variables de nivel macro tales como desempleo, crimen, educación y recursos. Enfoques de este tipo, como la dinámica de sistemas, habilitan para identificar retroalimentaciones y para ver los efectos a nivel de sistema sin empantanarse en detalles. Un micromodelo permite modelar procesos cognitivos o afectivos de actores individuales o como mínimo sus resultados - decisiones individuales y acciones- Los modelos a nivel micro incluyen los cognitivos de la psicología, los sistemas de expertos y la elección racional incluida la teoría de juegos y la teoría de decisiones (Zacharias, MacMillan y Van Hemel, 2008, p. 94). Dos son los tipos de mesomodelos, los de redes que permiten formalizar, medir y probar concepciones aproximadas de capital social, centralidad y conectividad; y los basados en agentes que permiten incluir agentes diversos y espontáneos que interactúan en espacio y tiempo. Es de advertir que dichos agentes pueden autoorganizarse, crear estructuras emergentes a nivel meso que asumen significado y tienen valor predictivo. Los modelos integrados vinculan las anteriores categorías (micro, macro y meso), recientemente, los investigadores comenzaron a crear modelos híbridos que incluyen agentes que emplean modelos psicológicos sofisticados y cuyos efectos macro se vinculan a un modelo de sistemas dinámicos (Zacharias, MacMillan y Van Hemel, 2008, p. 95).

Un sistema será sostenible en la medida que sobreviva y mantenga su integridad y 
estructura por un periodo de tiempo específico. En la modelación de sistemas complejos, escala y jerarquía son asuntos centrales. El mundo natural contiene una jerarquía conveniente de escalas de lo más simple a lo más complejo: de átomo, a molécula, a célula, a tejido, a órgano, a organismo, a población, a especie, a comunidad, a ecosistema y a bioregión. Cada escala resultante en este contexto, refiere la resolución (espacial, temporal o grado de complicación) y el alcance (en tiempo, espacio y número de componentes modelados) del análisis. Los fenómenos multiescala son particularmente predominantes en muchos sistemas de recursos naturales y muchas instituciones humanas. En la ecología y la economía, la información primaria y las mediciones son recopiladas generalmente en escalas relativamente pequeñas (pequeños terrenos en ecología, individuos o empresas en economía), a menudo dicha información es posteriormente usada para construir modelos en escalas radicalmente diferentes (Costanza, et al., 2001, p. 7).

Un sistema sostenible se caracteriza por basarse en el uso prudente de recursos renovables y reciclables; por proteger mediante regeneración continua de recursos la integridad de los sistemas naturales; por mejorar la calidad de la vida de individuos y comunidades; por ser rentable; y por estar guiado por una Ética de la Tierra que considera el bien a largo plazo de todos los miembros de la Tierra (Dinpanah y Lashgarara, 2008, pp. 6061). Con el fin de lograr la sostenibilidad y la protección del ambiente, han sido propuestos distintos modelos para contextos particulares. Por ejemplo, el Modelo Óptimo de Sostenibilidad (para Irán) de Dinpanah y Lashgarara (2008), sugiere que para prevenir la degradación de recursos naturales y del ambiente, entre otras cosas, se requiere 1) la creación de una red internacional de personal involucrado en el desarrollo y despliegue de soluciones prácticas para problemas reales de educación y sostenibilidad; 2) la identificación de buenas prácticas para ser compartidas y difundidas; 3) la transferencia, adopción y validación de buenas prácticas entre participantes, haciendo las modificaciones necesarias de acuerdo con particularidades locales; 4) la definición de nuevos paradigmas de educación en agricultura y recursos naturales que ubiquen la innovación y la sostenibilidad en sus entrañas y que reflejen las experiencias y aprendizaje de participantes; y 5) la instauración de un diálogo efectivo para facilitar la discusión y análisis de soluciones tangibles a problemas de sostenibilidad y educación. (p. 66). De igual modo, la Tragedia de los Comunes, el Dilema del Prisionero y la Lógica de la Acción Colectiva han sido modelos usados para interpretar la aún inacabada Teoría de la Acción Colectiva en torno a Recursos de Uso Común RUC como los naturales (Ostrom, 2000).

Bajo los anteriores referentes, el pensamiento sistémico resulta un enfoque apropiado para una investigación integrada sobre el DS resultando la interdisciplinaria la más adecuada. El principal objetivo de la investigación sostenible interdisciplinaria es el análisis de sistemas complejos con el fin de mejorar el entendimiento de conductas, incluidas las interacciones entre los componentes. Otro objetivo tiene que ver con la acción de dichos sistemas, para mejorar su eficiencia global además de su resiliencia. (Ghrab-Morcos, 2009, p. 686).

La tecnología siempre ha desempeñado un papel esencial en la prosperidad de las naciones. La aceleración del desarrollo tecnológico ha impuesto al sistema social y al natural, presiones externas tales que su respuesta y adaptación no les permite lograr un nuevo estado de balance en el tiempo exigido. El concepto de resiliencia ecológica emerge para caracterizar la habilidad de un sistema para adaptarse al cambio, para reorganizarse y regenerarse tras estar expuesto a turbulencias. Dado que el modelo de desarrollo actual resulta excesivamente consumista, es necesario repensarlo, surge por 
tanto el concepto de desarrollo sostenible centrado en la habilidad del hombre para ejercer el poder en formas diferentes. El objetivo del DS es entonces el desarrollo social y económico así como la prosperidad ambiental en el largo plazo, una suerte de crecimiento económico basado en equidad social y sostenibilidad en el uso de recursos naturales. Este concepto conduce a proponer modelos alternativos en tres dominios: producción, consumo y evaluación del desarrollo. (Ghrab-Morcos, 2009, pp. 684-685). La preocupación por la gravedad del cambio climático indujo un creciente interés en los modelos de producción eficientes en el uso de energía y de otros recursos, es bajo este modelo que aparece el concepto "cero emisiones". El ahorro de la energía por parte de las industrias no es suficiente, se demanda igualmente, un cambio en los estilos de vida; implica que la satisfacción de necesidades sociales se realice mediante soluciones menos intensivas en energía, en otras palabras modelos novedosos de consumo.

Para efectos de modelación es importante distinguir el sistema de recursos —áreas de pesca, cuencas subterráneas, áreas de pastizales, canales de riego, ríos, lagos, océanos y otros cuerpos de agua - y las unidades de recurso producidas por el sistema o individuos que se apropian o usan los sistemas de recursos — toneladas de pescado capturadas, metros cúbicos de agua que se extraen de un cuerpo de agua, toneladas de forraje consumidas por animales, cantidad de desperdicio biológico recibido por un río o canal. (Ostrom, 2000, pp. 66-67). Las unidades de recurso no se usan de manera conjunta, mientras que los sistemas de recurso sí.

\section{Impacto ambiental y modelo propuesto para la ciudad}

El impacto ambiental alude los "efectos o consecuencias de la incidencia humana sobre el medio ambiente o sobre sus componentes", su intensidad varía de acuerdo a las actividades que lo originan y las demandas para su recuperación (Martín y Santamaría, 2004, pp. 102). Es un hecho que para desarrollar actividades de tipo productivo, es necesario tomar del medio ambiente recursos naturales y que al ser procesados generan gran cantidad de residuos. La contaminación ambiental es una problemática generada por la actividad industrial y la cual se refiere a la "introducción directa o indirecta en la geosfera, atmósfera, hidrosfera o biósfera de sustancias, materiales o formas de energía, generalmente antropogénicas, que no forman parte de dichos ambientes o que están en concentraciones anormales produciendo un efecto perjudicial inmediato o futuro para uno o más componentes de los mismos" (Martín y Santamaría, 2004, pp. 46-47). Los residuos líquidos y gaseosos de plantas químicas, curtiembres, industria papelera y textil con altos contenidos de ácido sulfhídrico, contaminan el agua y el aire (Frers, 2005, p. 12). El agua residual de unidades industriales y comerciales, contiene contaminantes como sales, metales pesados, contaminantes orgánicos resistentes a la degradación que, al carecer de un tratamiento adecuado, causan serios problemas en la salud de las personas y en la preservación de las especies vegetales y animales. La contaminación de suelos se produce, entre otros, por la mala disposición y ausencia de tratamiento de las basuras y por el uso de pesticidas, herbicidas y fertilizantes que permanecen en el suelo y se integran a las cadenas alimenticias (Frers, 2005: 6). El asunto de suelos obliga no solo referenciar las actividades de contaminación de las empresas sino también las del uso inadecuado de la tierra que genera impactos ambientales.

Contaminación del aire. La contaminación del aire se da por emitir sustancias a la atmósfera como gases, polvos, olores y humos, que desequilibran su composición original (nitrógeno y oxigeno), atentando así contra la salud de las especies. Los contaminantes pueden ser de dos clases: los primarios, originados en procesos de combustión como el dióxido de 
azufre, el monóxido de carbono, el plomo y las partículas suspendidas; y los secundarios o tóxicos formados a partir de la reacción de los primarios con otros compuestos de la atmósfera como el ozono o el dióxido de nitrógeno. La presencia de contaminantes secundarios constituye uno de los principales problemas ambientales en las ciudades. En el aire bogotano se encuentran concentraciones de material particulado PM10 y PM2.5, de partículas suspendidas totales PST y de gases contaminantes como dióxido de azufre $\mathrm{SO}_{2}$, dióxido de nitrógeno $\mathrm{NO}_{2}$, monóxido de carbono $\mathrm{CO}$ y ozono $\mathrm{O}_{3}$.

Contaminación de aguas. Los cuerpos de agua de una ciudad incluyen los ríos, lagos, lagunas y canales. Los canales y ríos bogotanos reciben el agua residual de unidades industriales y comerciales como descargas de cromo, agua sanguinolenta, residuos químicos, residuos oxidados, basura y fibra de vidrio. Dichos vertimientos contienen contaminantes como sales, metales pesados y contaminantes orgánicos resistentes a la degradación que causan serios problemas en la salud de las personas y en la preservación de las especies vegetales y animales.

Contaminación de suelos. La contaminación de suelos se produce, entre otros, por la mala disposición y ausencia de tratamiento de las basuras pero en ciudades como Bogotá el asunto más serio lo constituyen los escombros. Los escombros generados por constructoras y por empresas públicas constituyen parte del paisaje en cualquier calle y elemento común del ornato público. No es extraño ver desechos de construcción y tierra, en separadores de puentes de avenidas o de autopistas, en zonas verdes o en cuerpos de agua de la capital colombiana. Aún más alarmante que escombros y basuras son los residuos peligrosos como baterías, aceites quemados, pilas usadas, desechos de químicos, grasas, pinturas, desinfectantes, tóxicos, inflamables y corrosivos, que son generados por el sector industrial y son dis- puestos de manera irresponsable en las afueras de la ciudad.

Si el desarrollo sostenible incluye las actividades que mejoran la condición humana y que pueden ser sostenidas en el tiempo, implica entonces minimizar el impacto ambiental. Entre las acciones contempladas figuran precaución y reducción de riesgos derivados de las actividades; protección y preservación del medio ambiente y de los recursos naturales y; garantía de las condiciones humanas actuales y futuras. Cualquier unidad social como puede ser el caso de una empresa será sostenible en la medida que use de forma prudente los recursos naturales, que proteja la integridad de los sistemas naturales y que mejore - $\mathrm{o}$ por lo menos no altere - la calidad de vida de las especies, incluida la humana. Aunque para efectos de modelación, ya han sido reconocidos y trabajados los tres asuntos (económicos, ambientales y sociales), en el presente apartado serán tratados de forma exclusiva los ambientales, pues en ellos se generan la mayoría de impactos sociales. Dado que ningún modelo ha sido considerado correcto y que el mejor enfoque depende de la naturaleza del problema a ser resuelto, aunado a que recursos naturales como aire, agua y suelos siguen vigentes para la explicación de dichos problemas, en esta ocasión y desde una perspectiva ostromniana se propone el modelo ambiental local de la figura 1.

La pretensión del modelo es encontrar mejores opciones en materia de políticas ambientales capaces de limitar el uso de recursos naturales en una ciudad capital como Bogotá. Obedeciendo a la recomendación de Ostrom en lo concerniente a modelación, se identifican los sistemas de recursos (atmósfera; humedales, canales y ríos; y espacios públicos) y las unidades de recursos producidas por las empresas que impactan dichos sistemas (material particulado PM10; Demanda Biológica de Oxígeno y Sólidos Suspendidos Totales; y escombros y residuos peligrosos). 
Figura 1

Modelo ambiental para Bogotá

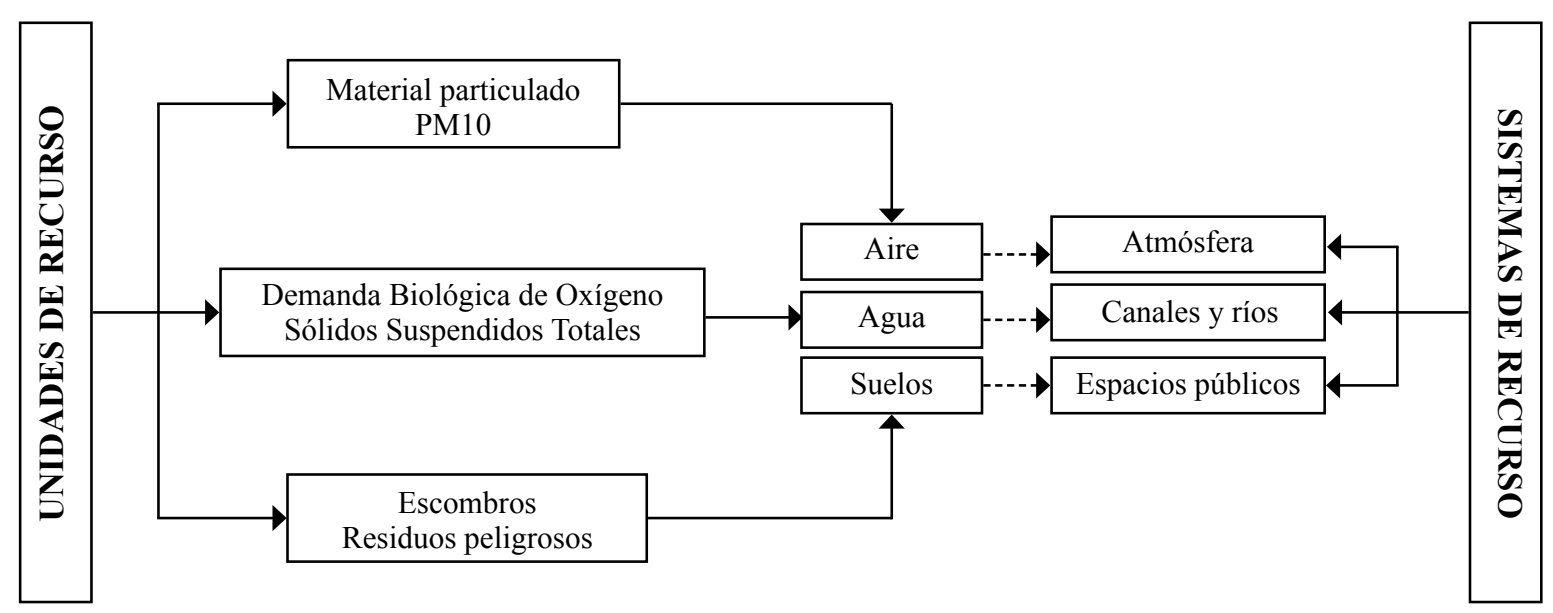

Fuente: Autores

Con este enfoque de modelación se persigue la identificación, compilación y relación de datos relevantes para entender los sistemas de recursos y tomar las decisiones que sean del caso. Si bien el análisis de los sistemas de recursos de la capital colombiana debe considerar las 20 localidades que la componen, aquellas que concentran las industrias son las que mayores impactos ambientales generan (Puente Aranda, Fontibón y Kennedy).

Aire. El principal contaminante de la ciudad es el material particulado compuesto por pequeñas partículas sólidas o líquidas de polvo, cenizas, hollín, partículas metálicas, cemento o polen, cuyo diámetro varía entre 2,5 y 10 micrómetros $(\mu \mathrm{m})$. La denominación PM10 refiere aquellas partículas que pasan a través de un cabezal de tamaño selectivo para un diámetro aerodinámico de $10 \mu \mathrm{m}$. Los componentes del PM10 son hierro, plomo, dióxido de carbono $\mathrm{CO}_{2}$ y polvo, de todos estos el de mayor presencia es el dióxido de carbono. Gran cantidad de éste gas proviene de la quema industrial de combustibles fósiles (carbón, petróleo y gas), de las plantas de poder y de los medios de locomoción. En Bogotá, el 75\% de la polución es atribuido al PM10 derivado del diésel y el restante $25 \%$ a las industrias, es de recordar que el $80 \%$ de las industrias bogotanas usan calderas de carbón emitiendo una concentración de 275 miligramos por metro cúbico de PM10 (las calderas de gas arrojan 7,5 miligramos). El promedio anual de PM10 en la atmósfera para 2007 fue de 2500 toneladas (Gómez, 2008a).

El propósito de la modelación del sistema atmosférico es conocer la dinámica de la polución ante cambios en las emisiones atmosféricas fijas y móviles pero también en procesos de siembra de árboles de la ciudad capital. La arborización contribuirá a la absorción de dióxido de carbono, uno de los componentes del PM10. En este sistema se identifican tres elementos (figura 2): la emisión de material particulado, el promedio anual de PM10 y la absorción de dióxido de carbono. Las relaciones establecidas "a más emisiones habrá más polución (positiva); a mayor absorción de $\mathrm{CO}_{2}$ menos polución (relación negativa); a más polución será mayor la emisión de contaminantes (relación positiva); y a más polución será mayor la absorción de $\mathrm{CO}_{2}$ (relación positiva)". 
Figura 2

Diagrama causal polución

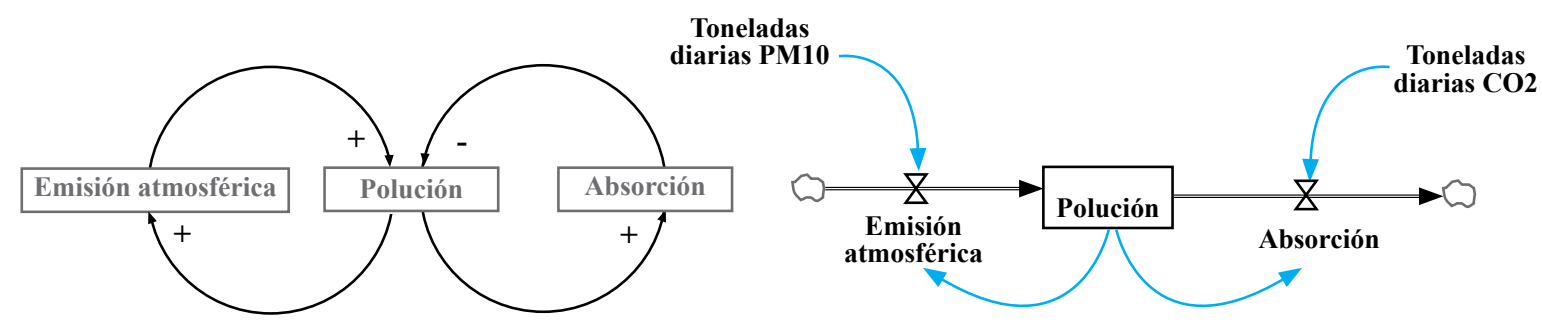

Fuente: Autores

El promedio diario de PM10 generado en Bogotá es de 13 toneladas por combustión de carbón y de siete toneladas por combustión de diésel (Gómez, 2008a). Los árboles y en general la vegetación son responsables de la absorción del $\mathrm{CO}_{2}$, según los estudios, un árbol sano y maduro absorbe seis kilogramos por lo que el inventario de árboles registrado en Bogotá (2007) y que asciende a 1.113 .765 árboles estaría en capacidad de absorber 6.683 toneladas de $\mathrm{CO}_{2}$ al año, es decir 18 toneladas diarias (Observatorio Ambiental de Bogotá).

Agua. En lo que a los ríos respecta, el Mapa de Vertimientos de Bogotá, permite identificar aquellas corrientes que mayor cantidad de vertimientos reciben, así como las respectivas fuentes contaminantes. Ríos como el Tunjuelo, el Fucha y el Salitre figuran como los más contaminados por los vertimientos de industrias y establecimientos comerciales. El Tunjuelo recibe lixiviados del Relleno Sanitario Doña Juana, descargas de cromo (250 kilos diarios) de las curtiembres de San Benito y agua sanguinolenta producto del lavado de las carnes y los locales de los expendios de productos cárnicos de la Autopista Sur. El Fucha recoge los residuos químicos de las tintorerías, los residuos oxidados, basura y fibra de vidrio de las chatarrerías del sector Montevideo y las aguas sin tratar de las industrias de Puente Aranda. El Salitre acoge residuos de 500 domicilios de las localidades de Chapinero, Teusaquillo y Barrios Unidos
(Gómez, 2008b). La calidad de los cuerpos de agua se mide a partir de la Demanda Biológica de Oxígeno (DBO) y los Sólidos Suspendidos Totales (SST). Las cargas DBO se definen como el oxígeno en masa (miligramos, gramos, kilogramos, toneladas, etcétera) que se requiere para degradar materia orgánica, tanto por la vía biológica como por la química, en cierto lapso de tiempo (minutos, horas, días o años). Los SST son partículas no solubles que no son lo suficientemente pesadas para sedimentarse en el cuerpo de agua en que están presentes, entre los principales sólidos figuran pequeñas partículas de materia orgánica e inorgánica, microorganismos y plancton (Observatorio Ambiental de Bogotá). La carga total de los ríos Salitre, Fucha y Tunjuelo en 2007 fue de 174.355 toneladas (Gómez, 2008b).

El propósito de la modelación del sistema de aguas es conocer la dinámica de carga contaminante frente a cambios en los vertimientos de materia orgánica e inorgánica y de residuos químicos y en la remoción de los mismos. En este sistema (figura 3) se reconocen tres elementos: carga contaminante en ríos, vertimientos de materia orgánica y sólidos, y remoción de sólidos. Las relaciones establecidas "a más vertimientos mayor carga contaminante (positiva); a mayor remoción de sólidos menos carga contaminante (negativa); a más carga contaminante serán mayores los vertimientos (positiva); y a más carga contaminante será mayor la remoción de sólidos (positiva)". 
Figura 3

Diagrama causal carga contaminante

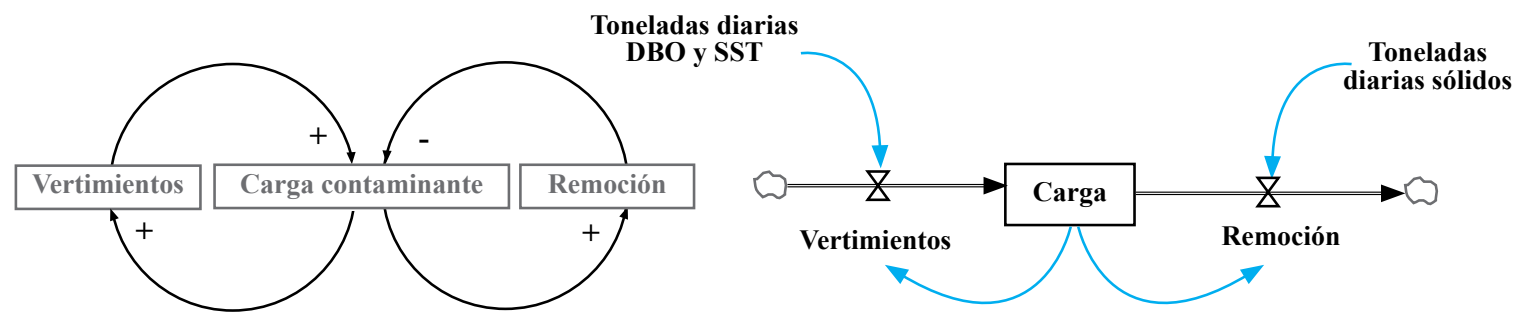

Fuente: Autores

La carga media diaria de DBO es de 327,73 toneladas y de SST es de 254,4 toneladas. Las plantas de tratamiento logran unas eficiencias de remoción del $40 \%$ de la materia orgánica expresada como DBO equivalente a 131,09 toneladas diarias y de $60 \%$ de los Sólidos Suspendidos Totales igual a 152,4 toneladas diarias (Observatorio Ambiental de Bogotá).

Suelos. Aunque las basuras constituyen uno de los sólidos dispuestos en los espacios no adecuados, son los escombros y los residuos peligrosos los verdaderamente alarmantes. Los escombros son los desechos de las obras de construcción, remodelación y/o demolición de la ciudad. Su naturaleza inerte y los grandes volúmenes generados son las principales características que afectan el posible manejo de estos residuos y están en gran medida determinados por los materiales y técnicas empleadas en la construcción (Observatorio Ambiental de Bogotá). El reducido número de escombreras operando en la ciudad (dos) su lejana ubicación y los costos de depósito son algunas de las causas de disposición de dichos residuos en espacios públicos o en rellenos sanitarios. Pero los escombros no sólo son generados por empresas constructoras sino también por empresas públicas como el Instituto de Desarrollo Urbano IDU, como la Empresa de Acueducto y Alcantarillado de
Bogotá EAAB y las empresas de servicios públicos (Secretaría Distrital de Ambiente, 2009). Por su parte y aún más preocupante, es la producción de residuos peligrosos (baterías, aceites quemados, pilas usadas, desechos de químicos, grasas, pinturas, desinfectantes, tóxicos, inflamables y corrosivos) y su posterior disposición en rellenos sanitarios o a cielo abierto. El $86 \%$ de este tipo de residuos es generado por el sector industrial y por lo regular su disposición final se realiza en municipios vecinos. El promedio anual de sólidos dispuestos de forma inadecuada es de 271.428 toneladas -258.133 de escombros y 13.295,10 de residuos peligrosos. (El Tiempo, 2008).

El propósito de la modelación del sistema de suelos es conocer la dinámica de la contaminación de suelos frente a cambios en la disposición de sólidos y en la recepción y manejo de los mismos. En este sistema (figura 4) se reconocen tres elementos: disposición de sólidos, contaminación de suelos y manejo y recepción. Las relaciones establecidas "a más generación de sólidos mayor contaminación de suelos (positiva); a mayor recepción de sólidos menos contaminación de suelos (negativa); a más contaminación de suelos será mayor la disposición (positiva); y a más contaminación de suelos será mayor la recepción de sólidos (positiva)". 
Figura 4

Diagrama causal contaminación de suelos

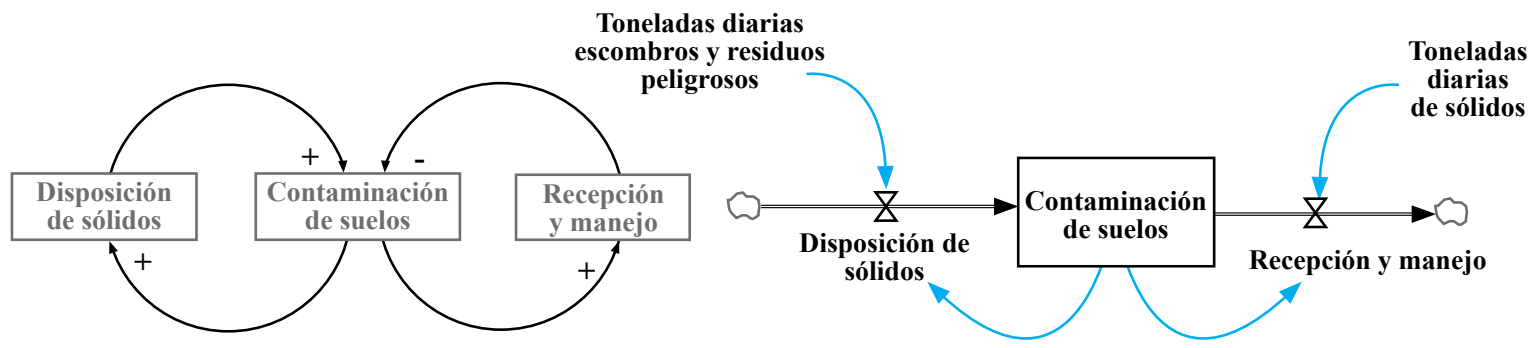

Fuente: Autores

El promedio diario de sólidos dispuestos indebidamente asciende a 751 toneladas (473 de escombros y 278 de residuos peligrosos). $\mathrm{La}$ capacidad de recepción y tratamiento de las escombreras que operan en la capital y de las empresas con licencia ambiental para el manejo de residuos peligrosos es de 205 toneladas diarias: 184 de escombros y 21 de residuos peligrosos (Secretaría Distrital de Ambiente, 2009).

\section{Simulación del impacto ambiental mediante lenguaje gráfico}

La modelación de sistemas dinámicos está aumentando, constituye una herramienta para: cerrar brechas espaciales y temporales entre decisiones, acciones y resultados; evaluar relaciones entre decisiones, acciones y resultados; y facilitar el aprendizaje cuyo requisito fundamental es que causa y efecto estén relacionados en tiempo y espacio. El principio fundamental de la dinámica de sistemas es que la estructura determina la conducta, es decir, que la forma como se separan los componentes de cualquier sistema, afectado y que afecta a los otros, determina la conducta emergente del sistema como un todo. (Brailsford, 2008, p. 1479).

Entre los diversos lenguajes gráficos de programación que facilitan la modelación de sis- temas dinámicos no lineales figuran: Dynamo, Ithink, Powersim, Stella y Vensim. Estos lenguajes de modelación además de definir inventarios, flujos, atributos e interacciones, especifican la capacidad de cuidado ecológico del sistema; el grado en que influencias externas son predecibles; la tasa de regeneración de los recursos; la tasa de transferencia de usuarios de recursos de una unidad espacial a otra; y la tasa de consumos de usuarios de recursos entre otros. A partir del apartado previo que identificó y estructuró, las relaciones de causalidad, los niveles, los flujos y las variables auxiliares, para cada recurso, se propone ahora, desde el lenguaje Vensim el modelo de la figura 5, quedando pendiente alimentarlo y correr la simulación.

Adicional a tener el acceso a la información demandada para alimentar el modelo, siempre será preferible tener participación en el diseño de la política ambiental para la ciudad. Una ciudad en la que la concentración de fábricas, automóviles, grandes complejos comerciales y residenciales es cada vez mayor. Asuntos estos que no solo afectan los recursos naturales (agua, aire y suelos) sino también la salud y bienestar de los residentes bogotanos. Es de aclarar que este modelo se inspira en el excelente trabajo de Shahgholian y Hajihosseini (2009) por lo que se recomienda su consulta directa. 
Figura 5

Modelo ambiental integral centrado en la producción

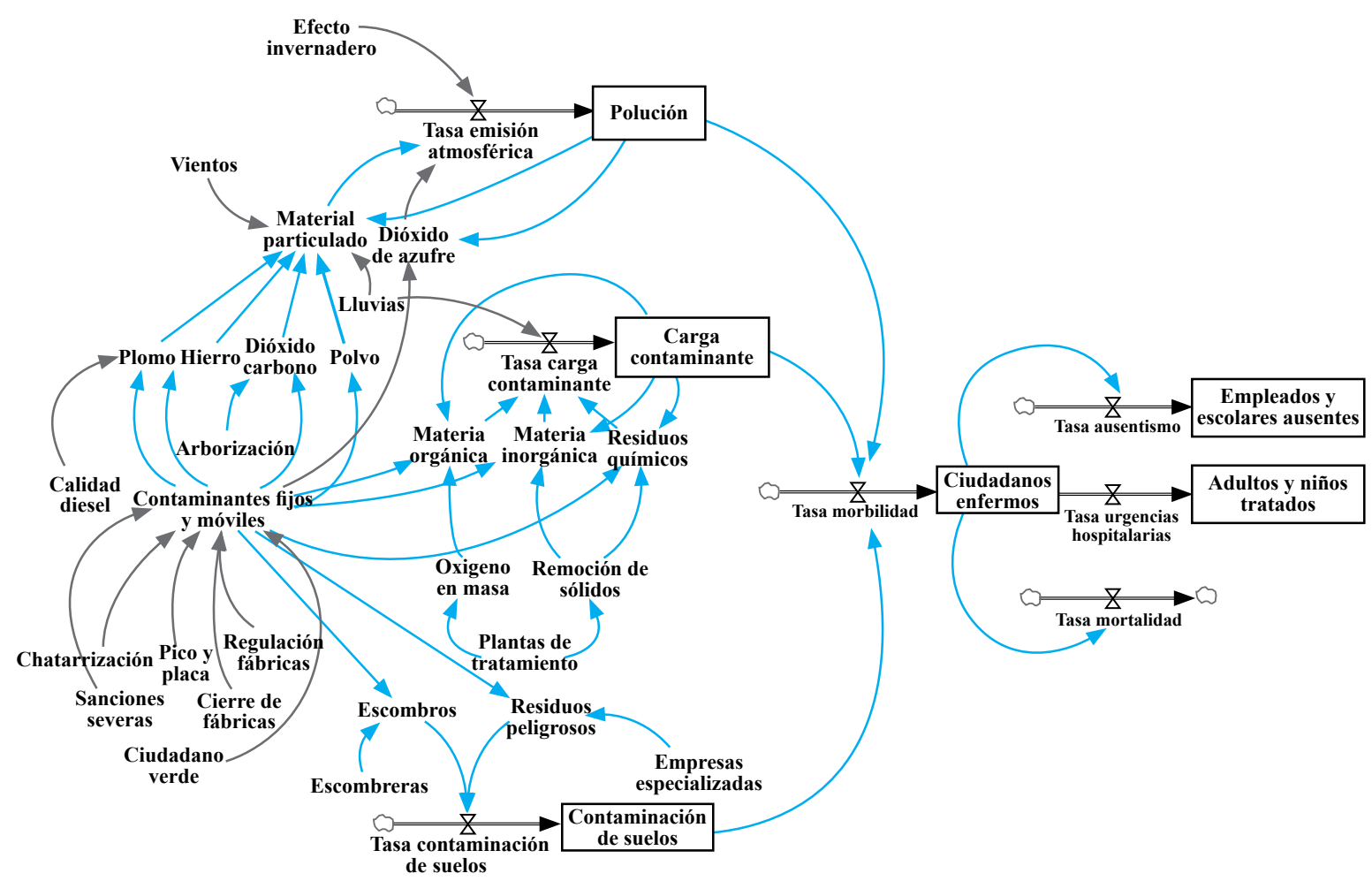

Fuente: Autores

Garantizando la información es posible la construcción de los indicadores de polución de carga contaminante y de contaminación de suelos, los cuales variarán en función de material particulado PM10, Demanda Biológica de Oxígeno y Sólidos Suspendidos Totales, y de escombros y residuos peligrosos, respectivamente. En los modelos propuestos, además de la siembra de árboles, de la disposición de plantas de tratamiento y al funcionamiento de escombreras y administradoras de residuos peligrosos (variables endógenas), deberán tenerse en cuenta otras variables (exógenas) como: comportamiento de los vientos, nivel de lluvias, efecto invernadero, calidad de diésel, regulaciones en circulación de automóviles (pico y placa), medidas de chatarrización de vehículos públicos, regulación de empresas contami- nantes, reubicación de fábricas, curtiembres y expendios cárnicos, imposición de sanciones (legales, económicas y morales) más severas y prácticas de consumo amigable, entre otras.

Sin desconocer que la polución, las cargas contaminantes en afluentes y la contaminación de suelos, afecta todas las especies de una ciudad (humana, vegetal y animal), el impacto en los individuos con el tiempo genera altos costos no solo para el gobierno distrital sino también para las empresas que contribuyeron a dicho impacto ambiental y desde luego para la sociedad en general. El modelo propuesto se centra en el impacto negativo sobre los bogotanos, dejando abierta la posibilidad para que otros investigadores, estudien y analicen el impacto sobre las especies animal y vegetal. 
Sin lugar a equivocación, en la medida que se logren reducir los indicadores de polución de carga contaminante y de contaminación de suelos, los efectos en materia social serán positivos. $\mathrm{Y}$ es que las tasas de morbi/mortalidad por enfermedades cardiacas y respiratorias agudas, de urgencias hospitalarias por infección respiratoria, de ausentismo escolar por enfermedades como gripa, bronquitis o neumonía $\mathrm{y}$ de ausentismo laboral por fuertes dolores de cabeza y molestias gastrointestinales; no son otra cosa que el resultado de que los elevados indicadores de polución y contaminación ambiental. Más grave aún, en función de la edad y del tiempo, el plomo respirado puede resultar cancerígeno o neurotóxico, producir trastornos en el comportamiento y dificultades en el aprendizaje, afectar los riñones y producir anemia. (Goméz, 2007, en Toca, 2011, p. 615). El modelo permitirá inferir los niveles de polución de carga contaminante y de contaminación de suelos para los próximos 10 años, la relación de cada uno de estos niveles con la población enferma y de ausentes laborales y escolares. De aquí, se podrá igualmente predecir los costos hospitalarios por concepto de tratamiento de enfermedades respiratorias, la productividad de las empresas, la eficiencia terminal de los escolares y los pagos de incapacidades por cuenta de las EPS, entre otros asuntos

Con los referentes expuestos y con los requisitos planteados, será posible concluir cuáles parámetros tienen mayores efectos sobre la polución, la carga contaminante y la contaminación de los suelos bogotanos. Mediante políticas distritales -centradas en regulación y cierre de fábricas por incumplimiento de la normatividad; pico y placa con finalidad ambiental, chatarrización oportuna de vehículos de transporte público; aumento de sanciones (legales, económicas y morales) a unidades productivas y familiares que impacten un recurso natural; mejora de la calidad del diésel y el fortalecimiento de la cultura de consumo verde- es muy probable que la calidad del aire del agua y de los suelos bogotanos mejore en el tiempo.

\section{Conclusiones y recomendaciones}

Si bien mediante acción colectiva y procesos de políticas reguladoras es posible garantizar resultados positivos para los recursos naturales, en Bogotá no opera la primera, por lo que la única opción es el gobierno de los recursos por la vía de las políticas públicas reguladoras. Dado que sobre fenómenos como los vientos y las precipitaciones - que contribuirían sobremanera a la recuperación de los recursos, especialmente aire y aguas - no se logra tener control, el gobierno distrital debe decidir sobre las variables controlables disponibles.

De manera concreta debe centrarse en: exigencia de tecnologías limpias en vehículos (convertidores catalíticos) y de filtros en las fábricas, demanda de mejor calidad del diésel distribuido en Bogotá, chatarrización de vehículos de transporte de pasajeros y de carga, reubicación de fábricas, disposición de planes de emergencia (Pico y Placa Contingente) cuando los índices de contaminación ambiental excedan los límites normales, planeación de arborización, incosteabilidad de las multas económicas por contaminación de recursos, invocación de la sanción social a empresas contaminantes (boicot y rechazo de sus productos), aumento de penas de cárcel por contaminación y condicionamiento de la aprobación de licencias de construcción (residencias, centros comerciales, etcétera) a la disposición garantizada de escombros, entre otras medidas.

\section{Referencias}

Brailsford, S. C. (2008). System Dynamics: What's in it for Healthcare Simulation Modelers. Proceedings of the 2008. Winter Simulation Conference. New York. Institute of Electrical and Electronics Engineers. 1478-1483.

Costanza, R, Bobbi, L, Ostrom, E, \& Wilson, J. (Eds.). (2001). Institutions, Ecosystems 
and Sustainability. Boca Ratón: Lewis Publishers.

Dinpanah, G, \& Lashgarara, F. (2008). Designing an Optimum Model for Protection and Improvement of Sustainability of Natural Resources and Environment in Iran. Annals of the New York Academy of Sciences, 1140, 60-67.

Duran, E. J. A, \& Paucar, C. A. (2009). System Dynamics Urban Sustainability Model for Puerto Aura in Puebla, México. Systemic Practice \& Action Research, 22, 77-99.

El Tiempo. (2008). 'Toneladas de residuos peligrosos generados en Bogotá van a parar a lugares sin ningún control'. Recuperado de http://www.eltiempo.com

Frers, C. (2005). Los problemas de degradar el suelo. Perú: Ilustrados.com

Ghrab-Morcos, N. (2009). Interdisciplinary Research on Energy: A Necessary Approach 0020 for Sustainable Development. Proceedings of World Academy of Science: Engineering \& Technology, 39, 684-690.

Gómez, L. (2008a). ' $80 \%$ de las industrias bogotanas usan calderas de carbón y emiten 13 toneladas de hollín al día'. Recuperado de http://www.eltiempo.com

Gómez, L. (2008b). Unos 994 vertimientos están matando los ríos Fucha, Tunjuelo, Salitre y Bogotá. Recuperado de http:// www.eltiempo.com

Martín, A. \& Santamaría, J. (2004). Diccionario terminológico de contaminación ambiental. España: Ediciones Universidad de Navarra.

Mueller, C. (2008). Sustainable Development: Conceptualizations and Measurement.
Brazilian Journal of Political Economy, 28(2), 207-225.

Mulder, P, \& Van den Bergh, J. (2001). Evolutionary Economic Theories of Sustainable Development. Growth and Change, $32,110-134$.

Observatorio Ambiental de Bogotá. Recuperado de http://oab.ambientebogota.gov.co/ porrecurso.shtml

Ostrom, E. (2000). El gobierno de los bienes comunes. La evolución de las instituciones de acción colectiva. México: UNAM, CRIM y Fondo de Cultura Económica.

Pew, R. \& Mayor, A. (Eds.). (1998). Modeling Human and Organizational Behavior: Application to Military Simulations. Washington: National Academies Press.

Sage, A. (1999). Sustainable Development: Issues in information, Knowledge, and Systems Management. Information Knowledge Systems Management, 1(3/4), 185-222.

Sahely, H. R, \& Kennedy, C. (2007). Water Use Model for Quantifying Environmental and Economic Sustainability Indicators. Journal of Water Resources Planning and Management, 133(6), 550-559.

Secretaría Distrital de Ambiente. (2009). 'Secretaría Distrital de Ambiente lanzó guía ambiental para el manejo de escombros'. Recuperado de http://www.secretariadeambiente.gov.co/sda/libreria/php/noticias08.php?id=1114

Shahgholian, K, \& Hajihosseini, H. (2009). A Dynamic Model of Air Pollution, Health, and Population Growth Using System Dynamics: A Study on Tehran-Iran (With Computer Simulation by the Software Vensim). World Academy 
of Science, Engineering and Technology, 59, 245-252.

Toca T. C. (2011). Desarrollo y calidad de la sociedad: compromiso del Estado y del mercado. Revista de Ciencias Sociales $R C S, 17(4), 612-624$.

Zacharias, G, MacMillan, J. \& Van Hemel, S. (Eds). (2008). Behavioral Modeling and Simulation: From Individuals to Societies. Washington: National Academies Press. 